\title{
Perspective
}

PERSPECTIVE Actualité en histoire de l'art

2| 2017

Le Maghreb

\section{Musée national/musée universel, musée global/ musée local : les musées de la rive sud de la Méditerranée}

Une discussion entre Moncef Ben Moussa, Meriem Berrada, Malika

Dorbani-Bouabdellah, Taher Ghalia et Abdellah Karroum, menée par Éric de Chassey

Moncef Ben Moussa, Meriem Berrada, Eric de Chassey, Malika

Dorbani Bouabdellah, Taher Ghalia et Abdellah Karroum

\section{(2) OpenEdition \\ 1 Journals}

Édition électronique

URL : http://journals.openedition.org/perspective/7476

DOI : 10.4000/perspective. 7476

ISSN : $2269-7721$

Éditeur

Institut national d'histoire de l'art

Édition imprimée

Date de publication : 31 décembre 2017

Pagination : 49-64

ISBN : 9782917902394

ISSN : $1777-7852$

Référence électronique

Moncef Ben Moussa, Meriem Berrada, Eric de Chassey, Malika Dorbani Bouabdellah, Taher Ghalia et Abdellah Karroum, « Musée national/musée universel, musée global/musée local : les musées de la rive sud de la Méditerranée », Perspective [En ligne], 2 | 2017, mis en ligne le 30 juin 2018, consulté le 01 octobre 2020. URL : http://journals.openedition.org/perspective/7476 ; DOI : https://doi.org/ 10.4000/perspective.7476 


\section{Musée national/musée universel, musée global/musée local : les musées de la rive sud de la Méditerranée}

\author{
Une discussion entre Moncef Ben Moussa, \\ Meriem Berrada, Malika Dorbani-Bouabdellah, \\ Taher Ghalia et Abdellah Karroum, \\ menée par Éric de Chassey
}

Cette table ronde ${ }^{l}$ relève d'une préoccupation ancienne : elle a d'abord été pensée dans le cadre d'un colloque sur les échanges culturels et artistiques entre les deux rives de la Méditerranée, dont la première session avait eu lieu à l'École du Louvre, à Paris, la seconde à la Villa Médicis, à Rome, et dont la troisième devait avoir lieu à Tunis au mois de juin $2015^{2}$ - avant que les attentats, à commencer par celui qui a visé le Musée national du Bardo le 18 mars 2015, faisant vingt-quatre morts, n'en décident autrement. Elle intervient aujourd'hui dans un contexte un peu différent : à mon arrivée à l'Institut national d'histoire de l'art, j'ai trouvé un projet de numéro spécial de la revue Perspective consacré au Maghreb. Régulièrement, la revue propose de faire un état de l'histoire de l'art dans un pays, en invitant aussi des chercheurs et spécialistes étrangers et, pour la première fois, Anne Lafont, alors rédactrice en chef, et Zahia Rahmani, conseillère scientifique du domaine "Histoire de l'art mondialisée ", avaient l'idée d'élargir cette invitation à un territoire plus large, celui du Maghreb, ce qui rejoignait mon idée de travailler sur les musées de la rive sud de la Méditerranée.

Vecteurs identitaires à forte charge symbolique, religieuse, politique, ou plus simplement économique, le patrimoine culturel et ses lieux de conservation constituent actuellement une cible privilégiée dans le climat d'instabilité faisant suite aux révolutions du "printemps arabe ». À la fois outils de diffusion de la connaissance de l'histoire de l'art, terrains pour des expériences individuelles des œuvres, lieux de constitution d'une communauté internationale à visée universelle et de représentation d'un état ou de construction d'une nation, les musées, incarnations de ces valeurs partagées, sont les scènes et les objets d'attaques sans précédents, dont la plus monstrueuse est sans doute celle qui a touché le Musée national du Bardo. Il s'agit, dans les pages qui suivent, de soulever la question d'un ou des musées universels, posée notamment par la déclaration sur la valeur des musées universels, signée en 2002 par quelques-uns des grands musées internationaux $x^{3}$ des musées de l'ancien monde, dont la conception de l'universel remonte aux $X V I I I^{e}$ et XIX $X^{e}$ siècles et s'avère très fortement liée à un projet colonial sans ouverture réellement globale. Peut-être l'ouverture d'un certain nombre de musées à vocation universelle, en particulier dans les pays du Golfe, change-t-elle aujourd'hui la donne.

Dans le monde globalisé qui est désormais le nôtre, caractérisé par la circulation des informations, des personnes et des œuvres, les musées - ceux qui existent depuis longtemps aussi bien que ceux qui sont en préparation, relevant aussi bien de décisions étatiques que d'initiatives privées -, tout en gardant des spécificités locales importantes,

\begin{abstract}
Moncef Ben Moussa
est docteur en préhistoire, archéologie, histoire et civilisation de l'Antiquité et du Moyen Âge de I'université Aix-Marseille. Archéologuecéramologue, maître assistant à l'université de Tunis, il est actuellement conservateur en chef du Musée national du Bardo et directeur du département des Musées nationaux à l'Institut national du patrimoine de Tunis.
\end{abstract}

Meriem Berrada conçoit et met en œuvre les projets artistiques de la Fondation Alliances depuis 2012. Elle a créé La Chambre Claire (2013),

un programme de soutien

à la photographie africaine émergente, et Passerelles (2014), un programme d'ateliers de sensibilisation à la création contemporaine en zones urbaines périphériques. Depuis 2016, elle pilote le développement du Musée $\mathrm{d}^{\prime}$ art contemporain africain Al Maaden (MACAAL) à Marrakech.

Éric de Chassey est directeur général de l'Institut national d'histoire de l'art et professeur d'histoire de l'art contemporain 
à l'École normale supérieure de Lyon. Il a publié de nombreux ouvrages sur l'art et la culture visuelle des $\mathrm{XX}^{\mathrm{e}}$ et $\mathrm{XXI}^{\mathrm{e}}$ siècles et a été commissaire de multiples expositions monographiques et thématiques en France et dans le monde.

Malika Dorbani Bouabdellah a été directrice du Musée public national des Beaux-Arts d'Alger de 1977 à 1994, après en avoir été conservatrice. Depuis, elle a notamment été collaboratrice scientifique au département des Peintures du musée du Louvre.

Taher Ghalia est archéologue et historien de l'art des

Antiquités classique et tardive, spécialiste du christianisme antique. Actuellement directeur du Développement muséographique à l'Institut national du patrimoine de Tunis et professeur associé à l'université de la Manouba, il a dirigé le Musée national du Bardo de 2003 à 2012 et mis en œuvre sa rénovation.

Abdellah Karroum a fondé en 2002 L'appartement 22 (Rabat), une structure dédiée à l'art contemporain, et les éditions hors'champs. Il est directeur artistique de l'Arab Museum of Modern Art de Doha (MATHAF) depuis 2013. se transforment radicalement. Des centaines, voire des milliers de musées se créent chaque année dans le monde, répondant à de nombreux modèles intermédiaires - et ce, immédiatement ou dans un second moment : ainsi voit-on des musées initialement créés dans le cadre d'initiatives privées devenir avec le temps des musées à vocation publique. Comment ces musées peuvent-ils être des espaces de construction du sens commun, particulièrement au sein de sociétés caractérisées par la tentation du repli sur un passé mythifié ou de la fusion dans une suspension de l'histoire transnationale ? Comment peuvent-ils articuler leur insertion dans le global tout en faisant place aux préoccupations et aux publics locaux? Comment ces lieux de mémoire contribuent-ils à la construction d'une narration historique dans un environnement arabo-musulman, intégrant de multiples façons des passés qui ne l'ont pas toujours été? L'idée d'un musée universel, au sens où il participerait de la constitution d'un sens commun qui aurait vocation à s'adresser à l'ensemble de l'humanité, peut-elle avoir un sens dans la situation qui est la nôtre? Quelle vision de l'histoire propose chacun de ces musées, qu'il traite ou rassemble des auvres avec une profondeur historique importante, ou qu'il soit consacré à l'art contemporain (ouvrir un musée est toujours une manière d'écrire une histoire, aussi pour demain)? À quel point ces questions historiques relèvent-elles ou non d'une forme de mythification? Autant de questions autour desquelles nous avons réuni des invités aux compétences reconnues [Éric de Chassey].

Éric de Chassey. Je propose que chacun commence par une brève présentation de l'institution qu'il représente en retraçant son origine, son histoire, son actualité, la façon dont elle relève de décisions étatiques ou d'initiatives privées, comment elle négocie ces différents aspects, en insistant à la fois sur le périmètre des collections qu'elle rassemble et présente, et sur la manière dont cette constitution de collections entre en résonnance avec une politique d'exposition temporaire, tout en gardant à l'esprit ce rapport entre le global et le local, qui sera le fil rouge de cette discussion.

Abdellah Karroum. Je suis très heureux d'être ici avec vous pour cette table ronde et je pense en effet que le Musée arabe d'Art moderne (MATHAF) de Doha, et L'appartement 22 de Rabat méritent en effet tous les deux de figurer parmi ce que vous nommez les institutions muséales de la rive sud de la Méditerranée. Ces deux structures sont des institutions en construction, encore très jeunes.

Le MATHAF se situe au Qatar, I'un des pays du Golfe, un pays très jeune aussi ayant gagné son indépendance au tout début des années 1970, grâce à l'industrie du pétrole qui a permis un développement et une production de richesses, comme une transformation sociale, au travers des correspondances, des voyages et des connexions avec les autres parties du monde. C'est une région qui a toujours été un lieu d'échange basé sur le commerce et, la notion de musée, si on l'envisage dans la perspective de l'objet, est toujours une relation à l'objet qui vient d'ailleurs : en avoir la curiosité, le posséder, le conserver, le transmettre. Le musée est né de l'initiative privée de Sheikh Hassan Al Thani qui, alors étudiant et amateur d'art, a commencé à collectionner des objets et des œuvres dans son palais, à voyager beaucoup, mais a constaté que dans son pays il n'y avait aucun musée, aucun lieu pour partager ces objets. L'envie de partager et de transmettre sa passion le conduit, en 1994, à proposer des résidences à des artistes du monde arabe et à les inviter à produire des œuvres. Quelques années après la deuxième guerre du Golfe, ces résidences accueillaient tous les artistes d'Irak ou du Soudan qui étaient en difficulté dans leur pays. Nombre de leurs œuvres ont été achetées et font aujourd'hui partie de la collection du musée, placée en 2004 sous la responsabilité de la Qatar Foundation pour la culture, la recherche et l'éducation. Le MATHAF se déploie, depuis 2010, dans une ancienne école de la zone intellectuelle 
et universitaire de Doha : la Cité éducative, où il est entouré d'un certain nombre d'universités internationales, avec des étudiants qui viennent du Golfe mais aussi du monde entier. Aujourd'hui le musée collectionne des œuvres issues de l'Afrique du Nord au Moyen-Orient, tout en développant un savoir autour de la collection, axé sur la question de la modernité et sur le monde arabe, et produit également un programme éducatif.

Meriem Berrada. Je suis en charge des projets culturels de la Fondation Alliances, une association à but non lucratif qui œuvre depuis 2010 pour le développement culturel au Maroc et en Afrique. Il y a un an nous avons développé le Musée d'art contemporain africain $\mathrm{Al}$ Maaden (MACAAL) à Marrakech. Ce musée, au-delà de la conservation des œuvres acquises depuis plus de trente ans par le collectionneur et mécène Alami Lazraq, qui est à l'origine du groupe immobilier Alliances, et de sa Fondation, entend promouvoir la création artistique africaine en favorisant son accès aux publics les plus larges possible. La collection compte aujourd'hui plus de 2000 œuvres qui ont plutôt été acquises par le mécène au gré de ses voyages et de ses rencontres, et on peut estimer qu'il conserve des œuvres dont les créateurs sont pour $70 \%$ des artistes marocains, pour $20 \%$ des artistes issus du reste de l'Afrique et, dans une moindre mesure, des artistes contemporains asiatiques, sud-américains, etc.... L'idée du musée dédié à la création africaine a germé dans l'esprit du collectionneur et mécène il y a un certain nombre d'années déjà, bien antérieurement à cet engouement récent pour I'art africain, et avant tout pour partager le plus largement possible l'expression visuelle de son époque. C'est une initiative totalement privée, portée aujourd'hui par son fils Othman Lazraq, actuellement directeur de la Fondation, qui a été nourri dès son plus jeune âge à cette passion et qui, avec les années, a aussi intégré lui-même de nouvelles œuvres et de nouveaux médiums (photographie, installation, vidéo) à la collection.
1. Vue de l'exposition «E-Mois : autobiographie d'une collection ", Marrakech, Musée d'art contemporain africain Al Maaden (MACAAL), mars septembre 2017.

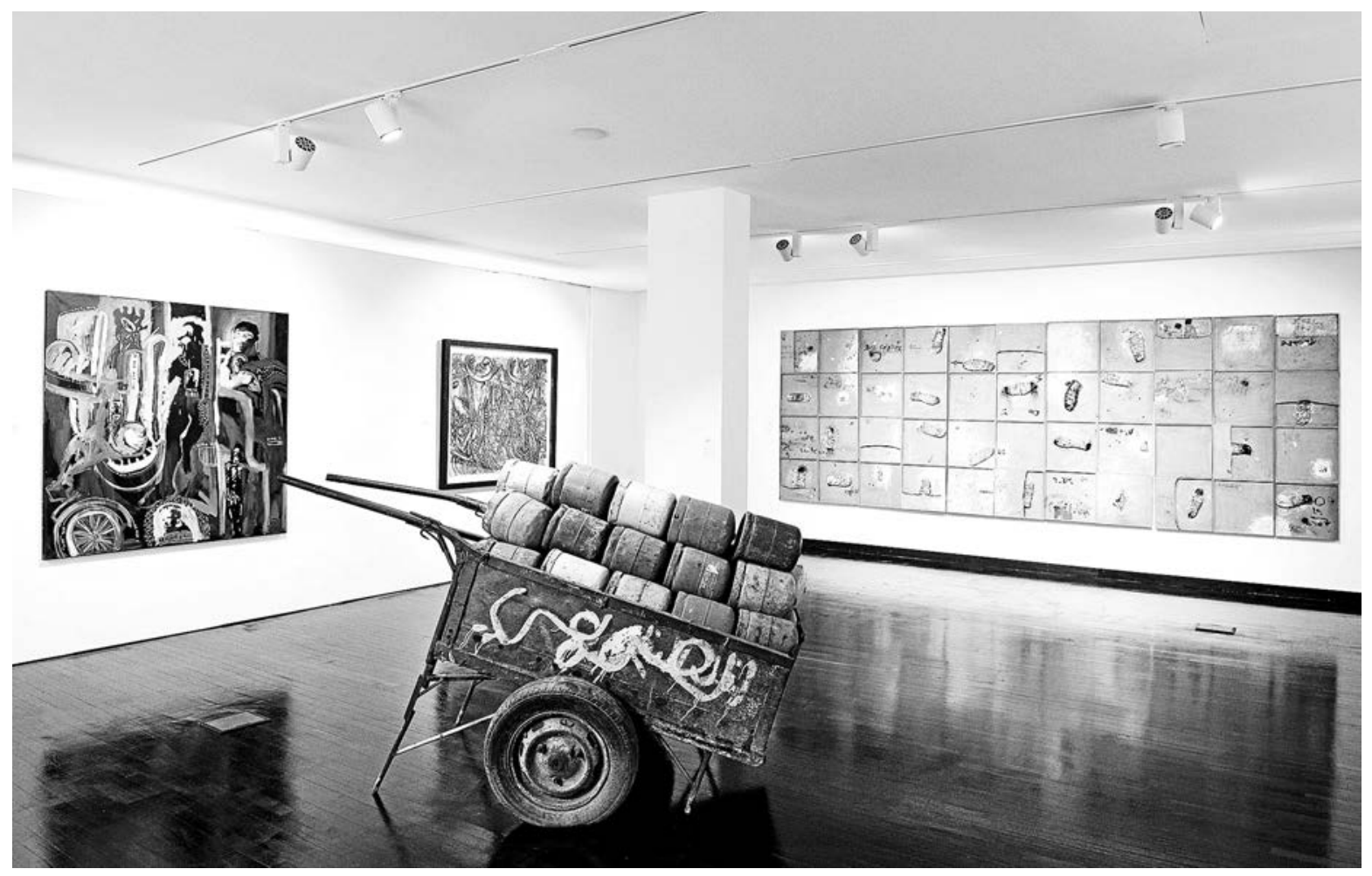


2. Stèle mosaïque commémorant I'inauguration du Musée national du Bardo, baptisé le " Musée Alaoui » en 1888.
Le rythme d'exposition du MACAAL est bi-annuel, et les deux expositions se déploient dans les $900 \mathrm{~m}^{2}$ d'espace d'exposition. Il y a un an, dans « Essentiels paysages ", au moment de la Cop $22^{4}$, l'idée était d'interroger une quarantaine d'artistes contemporains africains sur la question environnementale. Plus récemment, nous avons présenté l'exposition «E-Mois : autobiographie d'une collection», qui présente I'histoire de cette collection depuis sa naissance jusqu'à aujourd'hui (fig. 1). L'idée était de donner la parole à la collection qui se raconterait et s'adresserait au public elle-même en quelque sorte.

En termes d'écosystème le musée arrive dans le contexte d'une dynamique impulsée par le roi Mohammed VI, un grand collectionneur et un grand soutien pour I'art contemporain marocain : il est à l'initiative d'importantes manifestations valorisant la création contemporaine marocaine et, plus récemment, africaine. Le MACAAL est le deuxième musée dédié aux arts visuels au Maroc, après le Musée Mohammed VI d'art moderne et contemporain (MMVI) de Rabat, qui présente aujourd'hui davantage de blockbusters, comme une rétrospective consacrée à Alberto Giacometti ${ }^{5}$, les expositions "De Goya à nos jours. Regards sur la collection Banco de España $a^{6}$ », ou "Face à Picasso ${ }^{7}$ ", plutôt que des créations locales. Je pense, et cela n'engage que moi, que ces décisions étatiques tentent de suivre l'élan royal, et cela n'est à mon sens pas fait avec la vision, les outils et les ressources humaines nécessaires. Notre projet est apparu dans un contexte idéologiquement et symboliquement très favorable, mais pas structuré et nullement encourageant : nous agissons dans un cadre quotidien peu propice à l'action culturelle, avec peu d'aides à la création, une opacité des systèmes de subventions artistiques, un cadre fiscal inexistant pour les associations ou l'acquisition d'œuvres d'art, et je ne parle pas des taxes à l'importation d'œuvres d'art. Par ailleurs, les propositions artistiques émanent aujourd'hui en grande majorité d'initiatives commerciales privées, évidemment, qui n'intègrent pas du tout les enjeux de la médiation ou un discours servant à la construction d'un sens commun.

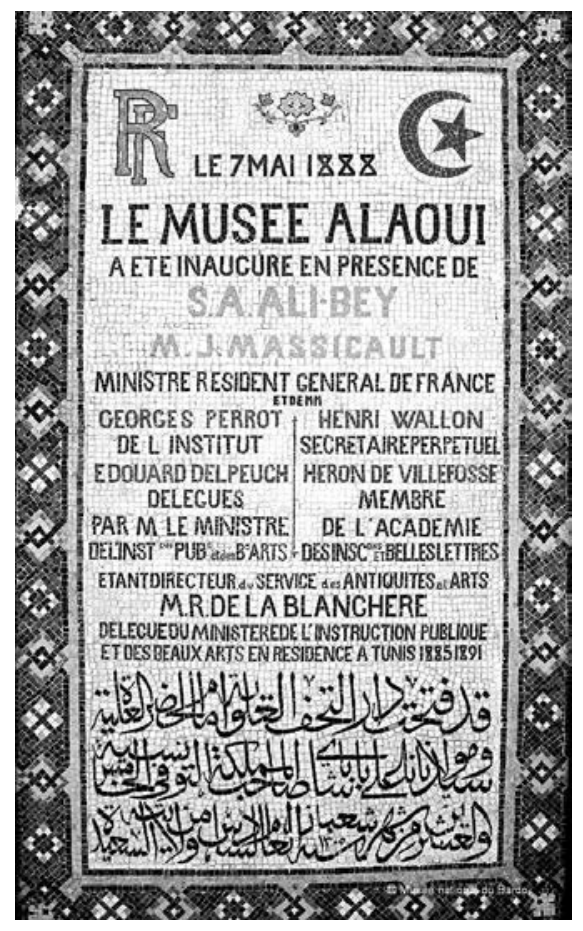

Moncef Ben Moussa. De par sa géographie, son histoire et les fondements de ses sociétés et de sa culture, la Tunisie est un musée à ciel ouvert. Le Musée national du Bardo est situé à la périphérie de Tunis, dans un quartier appelé le Bardo connu des voyageurs et des explorateurs. Hassan al Wazzan, dit Léon l'Africain, par exemple, le décrit comme le lieu « où sont les jardins et maisons de plaisance du Roi, fabriquées avec une architecture, non moins industrieuse, que superbe $[\ldots]^{8} »$. On sait que le noyau primitif de ce quartier fut construit sous le règne du sultan hafside Abou Fares Abd el Aziz al Mutawakkel (1394-1434). Centre du pouvoir husseinite depuis le début du XVIII e siècle (1705), Ali Pacha (1735-1756), second souverain de la dynastie, érige un premier palais dont l'escalier d'accès monumental est encadré par des lions. Hussein Bey, entre 1824 et 1835, se fait bâtir le «Petit Palais » tunisien de style andalou mauresque, puis, entre 1859 et 1864, M’hammed Bey construit le harem appelé Qasr Al-Badii, de style italianisant. Ces deux dernières demeures restent les résidences des beys jusqu'en 1879, lorsque Sadok Bey déménage dans le palais voisin, Qsar Saïd.

La reconversion de ce complexe palatial en un musée des antiquités nationales, annoncée par le décret du 25 mars 1885, est le résultat, entre autres, d'une politique (menée depuis 1876) du grand vizir Kheireddine Pacha qui réglementa la collecte des objets archéologiques et ordonna la saisie de la collection privée de $\mathrm{M}^{\prime}$ hammed Khaznadar, acquise frauduleusement. Depuis son inauguration (fig. 2), en 1888, le Musée national 
du Bardo, anciennement musée Alaoui, est une attraction particulière pour les Tunisiens et les visiteurs de la Tunisie. C'est le musée le plus important de la Tunisie, mais aussi du continent africain et du monde arabe, après celui du Caire. Avec le professeur Taher Ghalia, le musée a connu un grand projet de rénovation et d'extension (2009-2012 ; fig. 3). Les collections qui y sont exposées proviennent toutes des sites tunisiens et couvrent pratiquement toutes les périodes historiques. Il est vrai la période romaine est la mieux représentée, mais cela est dû à l'importance des fouilles et au nombre impressionnant des pièces archéologiques mises au jour dans la première moitié du XXe siècle.

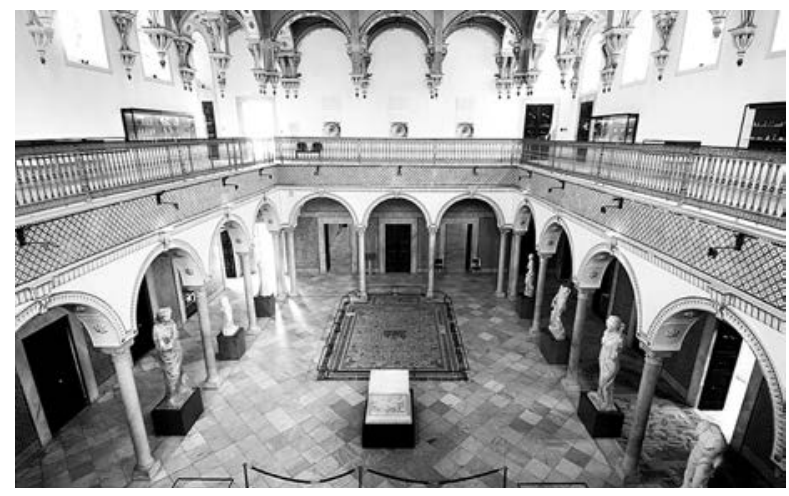

Éric de Chassey. Pour rebondir sur un aspect spécifique à la Tunisie, soulignons, par rapport à l'histoire des institutions patrimoniales, que nous sommes ici, sur la rive sud de la Méditerranée, en présence de l'un des rares cas d'existence d'une institution créée avant la période coloniale, mais avec un modèle qui ressemble fortement au modèle occidental de la tutelle des musées et des institutions patrimoniales. Autrement dit, il s'agit d'un modèle centralisé, avec un corps de responsables, dont l'inauguration fut suivie de créations d'institutions ailleurs sur le territoire. Pourriez-vous en dire un peu plus sur cet aspect avant que l'on nous abordions la situation algérienne?

Taher Ghalia. La Direction de l'antiquité et des arts, créée en 1882, devient, au moment de l'indépendance, l'Institut national d'archéologie et d'arts traditionnels, pour se transformer, en 1993, en Institut national du patrimoine (INP) - avec de larges prérogatives. La division du Développement muséographique, que je dirige, gère le quotidien de la totalité publics conservant des collections relatives au patrimoine matériel et immatériel. Elle octroie et gère leurs budgets, ce qui est énorme au vu des trente-cinq musées concernés. Avec la nouvelle loi des musées, rédigée il y a quelques mois, l'idée est d'aller vers une déconcentration. Le quotidien du musée tunisien est problématique aujourd'hui, et la priorité est de déléguer certaines prérogatives aux régions pour que les musées se transforment en entités culturelles qui peuvent faire leur travail. Le public tunisien est relativement peu présent et la fréquence des visites reste limitée ; il nous faut l'améliorer et l'augmenter, et il est désormais urgent de mettre en place une stratégie pour cela.

La centralisation date bien de l'époque du protectorat. Alors, le Musée du Bardo gérait tous les musées car c'était le seul à avoir été créé par un décret. Après l'indépendance, en 1966, il a perdu son autonomie, une mauvaise décision à mon avis car, étant donnée sa taille, ce musée devrait être autonome. Nous sommes maintenant en train de réfléchir pour trouver les meilleures solutions. Nous ne pouvons pas faire de sauts dans le vide, il nous faut des budgets. Pour octroyer un statut public d'établissement non administratif, il faut que les entrées soient visibles, que l'État accepte de donner une autonomie, et être autonome c'est aussi s'autogérer. Il faut donc que cela s'accompagne d'un travail important de formation des cadres, qui demandent à être qualifiés. Dans les musées tunisiens, une centaine de conservateurs travaillent, et il faut absolument professionnaliser ce corps qui a été surtout recruté après la révolution et mal réparti sur le territoire. Il faudrait encourager les universités tunisiennes à créer des masters professionnalisés dans les domaines de la gestion des musées, des expositions et des collections. Le véritable enjeu pour les musées en Tunisie est là, et nous attendons, avec la promulgation d'une loi des musées, un accroissement du nombre des musées publics et privés.
3. La salle de Carthage après la rénovation (2009-2012) du Musée national du Bardo, Tunis. 
Malika Dorbani-Bouabdellah. L'histoire du Musée national public des beaux-arts d'Alger commence, au XIX siècle, par des dépôts et des dons des musées français et du secrétariat aux Beaux-Arts, au profit des bureaux de l'administration civile et militaire et des édifices religieux. D'après le récolement de John Pradier de 1875, les critères de ces envois, des copies en général, manquaient de clarté. Les autres œuvres intégrées appartenaient à des collections privées, municipales, associatives et de sociétés savantes. Une partie d'entre elles est montrée au public, dans de mauvaises conditions de conservation, à la société des Beaux-Arts, créée vers 1860 par Laurent Laperlier, un ancien militaire et collectionneur, connu grâce au don de sa collection au musée Bonnat. C'est un constat de 1898 qui sert d'argument pour la création d'un musée digne des collections, et I'autonomie budgétaire vis à vis de Paris, obtenue en 1900, permet d'enrichir la collection et d'aménager, en 1908, un Musée municipal dans un local militaire. Un premier catalogue des collections paraît en 1911 sous la plume de Fritz Müller.

En 1920, le maire d'Alger désigne le musée comme facteur de cohésion sociale et d'identité. Une page de coran de Mohamed Racim entre en 1921 dans les collections - alors que le goût penche plutôt vers l'orientalisme, il s'agit bien de la première œuvre d'un artiste algérien à entrer dans une collection alors française. La préparation des célébrations du centenaire de la conquête de l'Algérie, le budget qui lui est alloué et surtout la personnalité de Jean Alazard permettent de rajeunir la collection en l'ouvrant aux artistes locaux, à la peinture nabie, fauve et post-cubiste, tout en suscitant de nouveaux dons, notamment de Frédéric Lung, de David-Weill et du baron Chassériauª. De musée municipal, le musée devient national. Il est doté de la personnalité civile, d'un bâtiment spécifiquement construit pour conserver et montrer des œuvres, d'une architecture inspirée de la muséographie moderne et du style néo-mauresque. Il est intégré, enfin, à une stratégie de développement touristique basée sur une originalité : donner un aperçu général de l'histoire des beaux-arts européens à dominante française et orientaliste, et alimenté par les œuvres des pensionnaires de la Villa Abdeltif et des artistes locaux.

Dans les années 1950, l'ensemble a une telle valeur qu'il est considéré comme partie intégrante des musées nationaux qui réclament un droit de regard : gestion, contrôle et inspection des dépôts de l'État notamment. La guerre de libération, la mort de Jean Alazard, les violences pendant les accords d'Évian, leur donnèrent gain de cause. Le rapatriement dont firent l'objet les pièces maîtresses, négocié et réglé par les accords d'Évian, est éloquent. La réclamation par les Algériens de ces œuvres est une preuve de leur volonté de patrimonialiser le legs français. Suite au protocole de 1968, qui détermine la propriété de chacun des protagonistes, les œuvres rapatriées sont restituées à I'Algérie en $1969^{10}$, sauf les pièces restées en dépôt au musée du Louvre. En échange, quelques œuvres des musées de France restent au Musée d'Alger.

L'accord relatif à la coopération culturelle a permis au Musée national des beaux-arts de reprendre vie en 1963 sous la responsabilité de Jean de Maisonseul, avec des œuvres d'artistes algériens, et d'accueillir, en 1964, les dons que les artistes de la Seconde École de Paris firent au «peuple algérien » pour qu'il se dote d'un musée d'art moderne et contemporain. Ce musée, le MAMA (Alger), existe depuis 2007, mais le vœu des donateurs reste pieux, leurs œuvres n'ayant pas été transférées. Jean de Maisonseul ayant quitté l'Algérie vers 1970, le musée a perdu sa personnalité morale et son public pour devenir une annexe du ministère de l'Éducation, de l'Information puis de la Culture. De Dar el ajeb (littéralement "maison de l'étrange », ou " des merveilles » ou « des miracles », « de l'émerveillement»), comme l'appelaient ses riverains, il fut considéré comme un « cimetière », un « tombeau », un "résidu colonial », « excentré »... autant d'identifications qui justifiaient l'inertie.

J'y suis arrivée en 1977 : je me devais de poursuivre l'œuvre de Jean de Maisonseul, de rappeler sa volonté de patrimonialisation, de lui rendre son prestige en tant 
que propriété de droit, en tant que patrimoine national, vivant, et de le considérer comme une chance, un « miracle» en appliquant les normes universelles de la muséologie. II a repris sa vie publique en 1982-1983 avec les expositions du fonds Racim et de " 10 ans de peinture algérienne » qui ont permis de reprendre le chantier des acquisitions là où mon prédécesseur les avait laissées. Il fallait aussi s'atteler à obtenir la personnalité morale, l'autonomie financière et

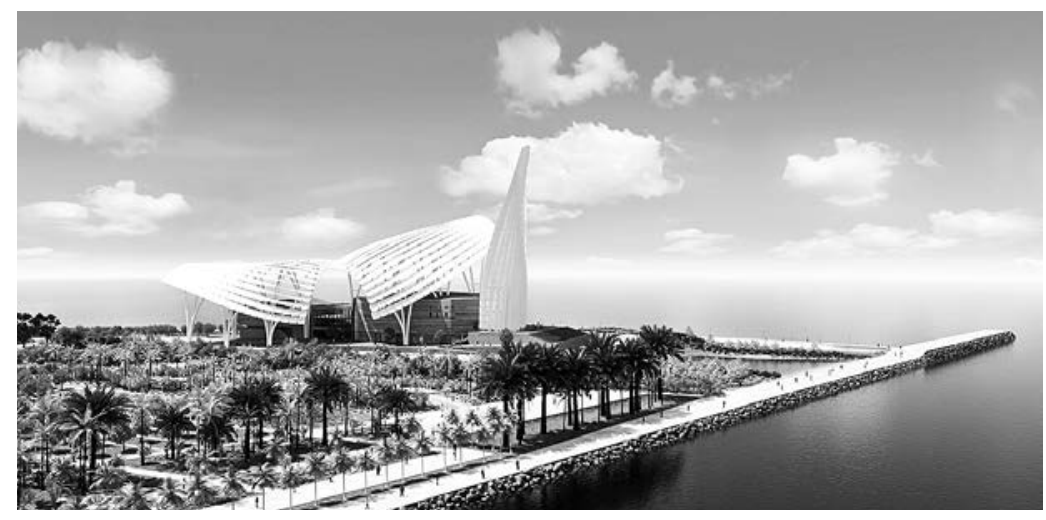
administrative du musée. En 1987,

sept autres musées nationaux sont nés, ce qui a permis d'établir un organigramme, une équipe, un programme, un budget, et dont l'effet le plus positif fut de pouvoir appliquer les normes de sauvegarde, de créer un service pédagogique, de poursuivre les acquisitions, de redonner vie à la revue et d'animer le musée. Cet élan fut brisé en 1994 et

4. Nadir Tazdaït mandataire et Pascale Langrand architecte associée, Grand Musée de l'Afrique à Alger, projet, 2016. relancé vers 1997. Il profite depuis de la vigilance nécessaire et des budgets alloués aux grandes manifestations culturelles et commémoratives organisées par les services de l'État et du ministère de la Culture.

L'année 2003, celle du programme " Djazaïr, une année de l'Algérie en France », a permis également de faire venir des artistes algériens en France, ainsi que les collections du Musée national des beaux-arts, notamment les dessins, au musée du Louvre ${ }^{11}$. Les années 2000 voient s'ouvrir de nouveaux musées, comme le Musée national d'art moderne et contemporain (MAMA) d'Alger, le récent musée d'Art moderne d'Oran (MAMO), ou encore le futur Grand Musée de l'Afrique (fig. 4). Les années 2000 ont donc été très fertiles pour les musées.

Éric de Chassey. L'un des éléments les plus frappants dans vos interventions est la complexité du rapport à la question nationale, négociée de façon extrêmement différente suivant les contextes. Depuis l'idée qu'aucun objet n'a sa place dans le musée s'il n'est pas issu du territoire national, d'une manière ou d'une autre, jusqu'à une vision plus lointaine, notamment celle qui intègre la patrimonialisation d'objets produits ailleurs, et qui rappelle un certain nombre de débats qui se déroulent en sens inverse dans les musées de la rive nord de la Méditerranée (sur la question des restitutions, notamment), et qu'il est important de poser aussi dans ce sens-là.

Nous savons que le musée comme forme à part entière est apparu dans un contexte contradictoire. Il apparaît traversé par une tension importante entre un discours de type nationaliste - le musée comme participant de la construction de la nation, y compris si l'on pense aux grands musées européens constitués vers les XVI'$X V I I^{e}$ siècles, quand la notion de "nation" n'existe pas en tant que telle, mais qu'il s'agit essentiellement de valoriser une famille régnante ou une personne privée qui aurait vocation à fédérer autour d'elle un ensemble géographico-culturel (ce qui pourrait rappeler un certain nombre d'initiatives qui existent dans les pays du Golfe) -, et une pensée de type universaliste. Si l'on relit aujourd'hui la déclaration de 2002 sur l'importance et la valeur des musées universels, on a du mal à ne pas y voir, de la part d'un certain nombre de grands musées occidentaux, une forme de protection, par précaution peut-être, quant à la justification de la nature d'une partie au moins de leur collection. Autrement dit, la constitution d'un discours sur la nation serait inséparable, 
à la fois, de sa projection dans l'ensemble du monde et, en même temps, d'une forme de revendication de l'universalité, qui serait mieux incarnée par telle nation que par telle autre. Or, le contexte que nous connaissons aujourd'hui est différent. Je pense en particulier à l'ouverture du Louvre Abu Dhabi, c'est-à-dire d'un musée à visée universelle, ou plutôt à visée globale, dans la mesure où l'idée même d'une visée globale remet en cause un certain nombre de fragmentations, de séparations traditionnelles à l'intérieur des musées - par département, par corpus géographique ou culturel. On a beaucoup écrit sur les contradictions de ce modèle depuis l'origine du projet. Récemment, dans un livre largement polémique, Alexandre Kazérouni ${ }^{22}$ en a tiré la conclusion qu'il existerait deux modèles de musées dans le monde arabo-musulman : le "musée racine " et le "musée miroir». Il ne laisse pas de place pour une alternative : soit un musée dont la légitimité serait liée à des "racines » nationales, et dont l'ancrage dans une histoire locale serait la seule et unique prétention, soit un musée qui accèderait à une forme de dignité ou de légitimité en se présentant comme un "miroir " de l'attente internationale de ce que devrait être un musée et, en d'autres termes, une manière pour un pays de jouer par là sa place dans le concert des nations.

Or la présentation des institutions que vous incarnez montre qu'il existe bien des alternatives. En particulier, j'observe un emboîtement en forme de poupées russes, qui n'existe pas dans les musées traditionnels de la rive nord de la Méditerranée, entre le local, le national et quelque chose d'autre qui, suivant les cas, serait l'Africain, le niveau continental ou régional, l'Arabe (ce qui n'est pas seulement une question géographique), et enfin un dernier niveau qui serait l'universel ou le global.

Moncef Ben Moussa. Le Musée national du Bardo est un musée très particulier, un lieu de contradictions, y compris dans son appellation : après 1907, à l'époque où il avait son autonomie, nos ancêtres l'appelaient Dar el ajeb, comme le musée d'Alger, or on sait que lors de son inauguration, il avait été nommé « musée Alaoui », du nom d'Ali Bey (1882-1902), et que désormais, depuis I'indépendance, on a I'habitude de parler du « musée du Bardo ». Coexistent ainsi cet attachement, cette préoccupation de lui donner un aspect local (le Bardo est le nom du quartier) et ce besoin de le considérer comme un musée proprement tunisien.

Or, que ce soit à travers ses collections, son appellation ou encore la perception de son rôle, les deux discours national/universel ont toujours été inséparables. Même lorsqu'ils s'expriment séparément ils se complètent car, à travers les collections locales, on aperçoit aisément une dimension culturelle méditerranéenne, voire universelle. Dans l'évolution historique retracée, les trois millénaires de la période historique sont représentés à travers les différents départements et les différentes cultures : punique, hellénistique, romaine, byzantine, arabo-musulmane et moderne. Les chefs-d'œuvre artistiques de ses collections permettent une immersion dans les mœurs et les activités quotidiennes, les loisirs, la vie sociale, les activités économiques ou encore le sentiment religieux. Ils conduisent à aborder des thèmes qui, sans être spécifiques au pays, reflètent, au-delà de leur dimension humaine, la diversité des racines et des composantes de ce qu'est la culture tunisienne aujourd'hui, dont l'unicité et la spécificité identitaire sont constituées par l'épaisseur des sédiments laissés par chaque culture que le sol tunisien a vu naître, se développer, s'articuler et fusionner dans le temps.

Taher Ghalia. Il faut ajouter que la Tunisie a été précurseure dans le monde des musées parce qu'elle est entrée très tôt dans la modernité : il ne faut pas oublier l'abolition de I'esclavage, qui date de 1857, la constitution en 1861, et le rôle d'Ahmed ler (bey de Tunis de 1837 à 1855). Ayant vécu en France, il voulait faire un musée à l'image du Louvre, mais il se souciait aussi de l'artisanat tunisien ; il souhaitait donc valoriser le 
patrimoine à Tunis, ce qui est une idée très intéressante. Après l'indépendance, une génération de chercheurs, d'ethnologues et d'archéologues tunisiens a effectué un travail considérable. C'est un phénomène d'appropriation du patrimoine national dans sa diversité. On appelait le Musée du Bardo Dar el ajeb parce que les Tunisiens s'étonnaient, parce qu'ils n'étaient pas conscients que l'antiquité et ses cultures faisaient partie de leur histoire - et la France ne faisait rien pour le leur expliquer, les arts et traditions semblaient bien suffisants. À présent, il faudrait penser à créer les conditions nécessaires pour que le public du monde musulman se réapproprie son patrimoine. Et je pense aussi au patrimoine immatériel : il faut le muséaliser, c'est comme cela que l'on va donner un second souffle aux musées dits « des arts et traditions » qui sont, qu'on le veuille ou non, d'inspiration orientaliste.

Malika Dorbani-Bouabdellah. En Algérie, je pense qu'on en est encore au « roman » national. Par opposition, le " récit » exige raison, savoir, démonstration par la preuve, débat, liberté de conscience et d'expression. L'histoire nationale serait une science, une école de jugement, capable d'aider les gens à devenir des individus, des citoyens qui s'estiment et qui sont solidaires d'autrui. Comment enseigne-t-on et expose-t-on I'histoire ? Les périodes qui la constituent sont-elles enseignées et expo-

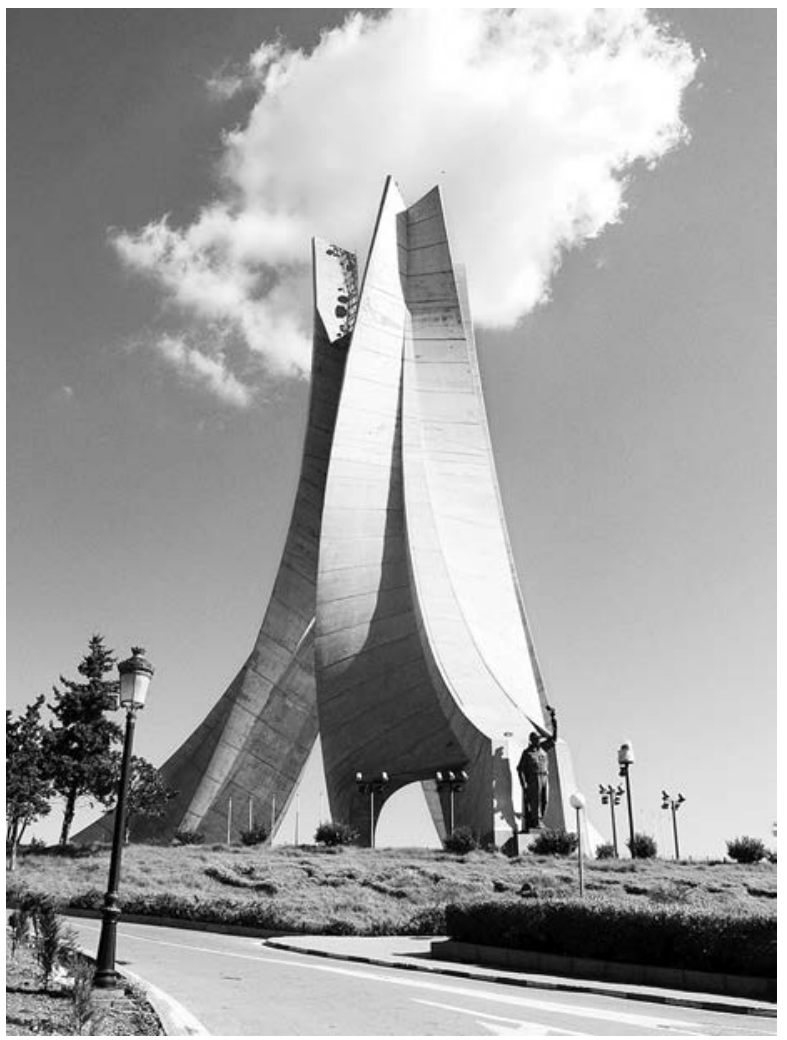
sées sans hiérarchie de valeurs, en rapport les unes avec les autres, de manière à la dépassionner, la dédramatiser, à créer un environnement serein ? II y a encore aujourd'hui des ruptures et des ignorances défavorables à l'émergence d'un récit proprement dit.

L'Algérie est dotée d'un réseau de musées où se trouvent les éléments constitutifs d'un récit national : préhistoire, antiquité, art musulman, ethnographie, arts et traditions populaires, beaux-arts, art moderne et contemporain, guerre de libération ou militaire, lieux de mémoire, sites archéologiques et historiques, monuments (fig. 5), symboles, faune, flore, etc... Mais il est tronqué et ne construit pas de relations manifestes entre ces différents substrats. Ce qu'il faudrait pour constituer un récit national cohérent c'est un musée $d^{\prime}$ histoire où seraient concentrés les repères essentiels, et qui bénéficierait d'une politique de mouvement et d'acquisition rationnelle des collections, de manière à donner un sens, une forme, à l'identité, à l'unité, à la vitalité, à l'imaginaire, à tout ce dans quoi se reconnaîtrait la majorité pour former une communauté nationale solidaire des autres peuples, des autres sociétés, des autres cultures car il n'y a qu'une civilisation, celle de tous les hommes.

Abdellah Karroum. Restreindre la définition du musée par l'alternative " miroir » ou « racine » me semble en effet très limitatif, c'est sans doute une idée qui concerne une projection propre et non la réalité des musées. Moncef Ben Moussa a très bien défini la Tunisie comme un musée à ciel ouvert, et beaucoup de parties du monde peuvent être perçues comme des musées à ciel ouvert. De même les notions de collection, d'échange - que l'on pense au commerce qui se déployait dans l'Océan indien, par exemple, et aux échanges de textiles, de perles, d'objets d'art, etc... -, tout comme celles d'héritage, de patrimoine, ne sont pas seulement européennes.

5. Bachir Yellès (d'après), Mémorial du Martyr, monument aux morts érigé en 1982, à l'occasion du $20^{\mathrm{e}}$ anniversaire de l'indépendance de l'Algérie, Alger. 


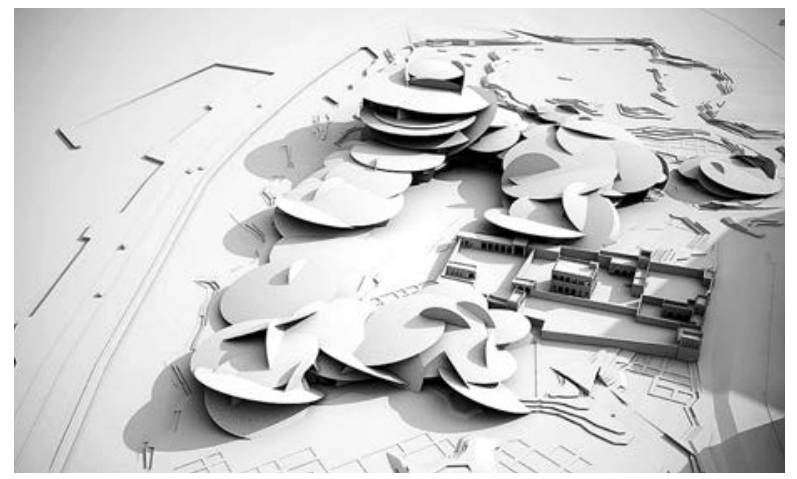

La relation à l'objet, à l'environnement et au passé a toujours existé, sous des formes qui impliquaient une conservation et des relations à notre environnement différentes. À propos de cette notion de musée national ou universel, je pense que le modèle d'Abu Dhabi, par exemple, est d'abord le développement d'un projet d'expansion urbaine et de développement touristique pour la ville du futur. Le manque de temps fait que l'on importe les grands musées qui existent déjà, le Louvre, le Guggenheim. Dans les pays voisins, on trouve une situation très différente, on construit un musée national. Il est d'ailleurs intéressant de noter que les musées du Louvre Abu Dhabi et du Qatar sont tous deux construits

6. Jean Nouvel - Ateliers Jean Nouvel, projet pour le Musée national du Qatar, Doha, 2008 - chantier en cours. par Jean Nouvel. Pour le Musée national du Qatar, il conçoit un bâtiment qui est une rose des sables (fig. 6), il part d'un objet local, naturel, du paysage, tandis que le projet muséographique est conçu avec les habitants. Le parcours part de I'histoire géologique, quelque chose de très universel comme l'histoire naturelle, puis intègre toute une partie de l'histoire orale, sociale et culturelle, enfin le musée lui-même, avec des galeries qui correspondent à des époques historiques, ponctuées par des vidéos d'artistes contemporains. C'est une pensée humaine qui construit un espace de vie, un espace de partage et, en tant qu'être humain, on est d'abord en relation avec l'espace, avec la perception sensorielle de l'environnement. L'expérience du musée peut être assez similaire quand on part du sensible; $c^{\prime}$ est l'interprétation qui change d'un pays à I'autre, d'une langue à une autre. II ne faut pas oublier la dimension sensible du musée comme contexte, elle est fondamentale. Et chaque lieu peut avoir son propre musée. Même si ce n'est plus le cas aujourd'hui, la vision européo-centrée d'une histoire de l'art universelle a pendant trop longtemps été celle d'une histoire de l'art de l'Europe, complétée par quelques figurants. J'aime beaucoup l'expression de Okwi Enwezor, lorsqu'il parle d'un " multiply located offcenter ${ }^{13}$ »; de ce point de vue chaque culture est le centre du monde globalisé.

Meriem Berrada. Je vais parler d'appropriation aussi, même si je pense en effet que ce double paradigme national/universel est toujours d'actualité. Il y a un véritable désir d'affirmation pour nous, d'une part parce qu'à travers ce musée nous cherchons à montrer autre chose de l'Afrique que ce que dépeint l'actualité économique, politique ou sanitaire et que, d'autre part, dans cette affirmation, il y a aussi l'idée de montrer que cette histoire nous appartient. II y a effectivement la question de ce qu'il reste de la colonisation : des histoires, des vécus sans doute différents selon la durée de la présence coloniale, la situation géopolitique, etc., mais aussi un dénominateur commun. Et c'est en partie pour répondre à cette question que le musée définit son objet comme l'art africain.

En même temps, le MACAAL se trouve incontestablement investi d'une ambition d'universalité qui se reflète dans l'ouverture de notre structure à l'échelle internationale, ainsi que dans sa mise en réseau sans distinction d'origine géographique. Nous sommes en effet aussi bien partenaires de l'École supérieure des arts visuels de Marrakech que de l'Art Club International The Cultivist, de la Biennale de Bamako ou encore du groupe de collectionneurs Sotheby's Preferred. Notre ambition se retrouve aussi quotidiennement à travers la sélection des œuvres acquises et exposées ainsi que dans notre programmation, afin d'éviter de tomber dans le piège du cliché. Ce souci est partagé par les artistes également, dont certains refusent d'être identifiés comme étant Maghrébins, Africains, etc. 
Permettre une appropriation est une volonté claire du mécène, parce qu'en réalité l'histoire de l'art au Maroc reste marginalement connue, des noms comme Chaïbia Tallal ou Farid Belkahia, récemment disparu, sont finalement de pures abstractions pour la plupart des Marocains. La collection s'adresse aux visiteurs, sans intermédiaire, elle est une invitation à l'observation, au questionnement et à l'appropriation. Ce qui sous-tend cette démarche est évidemment une forme de nationalisme. S'appuyer sur ce sentiment, dans notre cas, c'est lutter contre l'exclusion culturelle d'une partie de la population, dire qu'il ne s'agit pas de l'art d'une élite, de l'art d'un Maroc lointain, mais de votre art, votre expression, votre nation et, plus récemment, votre continent. Nous avons monté en 2013, par exemple, un programme destiné à la photographie émergente, avec pour ambition de produire la première exposition personnelle $d^{\prime}$ un jeune photographe. Nous l'accompagnons dans toute la démarche de création et de rencontre avec le public, et nous allons chercher des jeunes issus des quartiers périphériques de Casablanca qui viennent visiter l'exposition en compagnie de l'artiste et de médiateurs. Les enfants du quartier de Sidi Bernoussi étaient venus pour voir I'exposition dans les locaux de la Fondation Alliances; puis nous avons voulu faire voyager l'exposition, qui a été reçue dans un centre d'art d'un quartier qui leur est proche. Les mêmes enfants sont revenus voir l'exposition et $\mathrm{m}^{\prime}$ ont dit : " Maintenant C'est notre artiste. »C'est un symbole qui paraît fort parce que l'art ne fait pas partie de notre quotidien, et les arts visuels sont, à l'échelle internationale, encore quelque chose de très élitiste. Nous portons une volonté de démystifier tout cela.

Éric de Chassey. Situés dans des pays marqués à la fois par leur insertion, souvent violente, dans des empires plurinationaux (empire romain, califats de la conquête arabo-musulmane, empires perses, empire ottoman, empires coloniaux européens), par la conquête de leur indépendance et par le rassemblement en leur sein de communautés plurielles (présentes depuis des siècles ou arrivées récemment), les musées que vous représentez vous semblent-ils devoir ou pouvoir refléter de façon exhaustive cet héritage? Peuvent-ils contribuer à la construction d'une narration historique dans l'environnement arabo-musulman, qui pourrait intégrer - et de quelles façons des moments du passé ou une création artistique présente qui ne le sont pas toujours?

Enfin, comment les musées et les institutions culturelles de la rive sud de la Méditerranée peuvent-ils être des lieux de construction d'un espace et d'un sens communs, particulièrement au sein de sociétés caractérisées par la tentation du repli sur un passé mythifié ou de la fusion dans une suspension de l'histoire transnationale? Quelles politiques d'éducation ou de médiation mettent-ils en place pour accueillir et/ ou former les publics locaux? Quelle exemplarité peuvent-ils représenter pour une scène internationale des musées caractérisée par de nombreuses remises en cause et doutes, en particulier face aux pressions du système marchand et du tourisme de masse?

Meriem Berrada. Dans le cas du MACAAL, je pense que cela se fait naturellement, car présenter la création du continent, c'est montrer son histoire. Il ne s'agit donc pas d'une position militante pour nous, bien que notre programmation et nos expositions dépeignent d'elles-mêmes cette histoire multiple et complexe. Cette identité plurielle se retrouve dans le préambule de la Constitution marocaine ${ }^{14}$. En cela, nos musées contribuent à la construction d'une narration historique intégrant parfaitement des épisodes du passé comme la création artistique présente. Cependant, je nuancerais la question concernant I'environnement arabo-musulman, qui n'est pas véritablement synonyme d'unité.

Concernant la seconde partie de votre question, à mon sens, il s'agit d'un devoir. En effet, les musées sont parfois les seuls réceptacles d'expressions et de discours 
7. Dédicace de la salle de prière provenant de la synagogue de l'antique Naro (Vle siècle), découverte en 1883 à Hammam Lif, Tunis, Musée national du Bardo. pouvant faire contrepoids aux replis et aux conservatismes. Je pense notamment au développement d'un centre culturel dans le quartier défavorisé de Sidi Moumen de Casablanca, initié par l'écrivain et artiste Mahi Binebine et le réalisateur Nabil Ayouch, qui propose des ateliers de danse, d'arts visuels et de musique de grande qualité en réponse aux actes terroristes de Casablanca en 2004 dont les auteurs étaient issus de ce même quartier. À travers ce type d'initiatives, il ne s'agit pas de revendiquer une histoire nationale, mais plutôt d'aller dans le sens d'une ouverture culturelle large, là où le système éducatif ne remplit pas sa mission. La pertinence de ce type $d^{\prime}$ initiatives réside aussi dans le refus de penser en termes de modèles préétablis. Chaque projet doit s'envisager en fonction de son environnement, avec une vision claire de celuici, tout en tenant compte des spécificités de ses publics. En termes d'exemplarité, le MACAAL ayant fatalement affaire à des publics moins " éduqués ", fait particulièrement prégnant au Maroc, nous nous concentrons davantage sur des outils de médiation comportant peu d'écrit. En effet, il est important de rappeler que 32\% de la population marocaine est encore analphabète, que le taux d'élèves parvenant au baccalauréat est également faible, que le système scolaire public est complètement défaillant et les langues d'usage ne sont pas parlées uniformément par toute la population. Ainsi, certains médiums autres que l'écrit peuvent être une voie à privilégier au sein des parcours d'exposition.

Cette conviction préexistait au MACAAL, étant donné que j'avais initié en 2013, avec mon collaborateur en charge de l'axe social de la Fondation, le programme "Passerelles ${ }^{15}$ ", une démarche visant à sensibiliser des jeunes de zones périurbaines de Casablanca aux arts visuels. Plus récemment, dans la lignée de sa volonté de transmission, de partage et de diffusion de l'art contemporain africain, nous avons initié le MACAAL Lab, un espace visant à la valorisation des pratiques artistiques contemporaines. Une salle du musée spécifiquement dédiée au programme se trouve ainsi investie par l'œuvre d'un artiste contemporain africain dont la pratique inclut l'utilisation des nouveaux médias. Ces focus sont chaque fois accompagnés d'un programme de workshops en présence de l'artiste autour du processus de création de l'œuvre, de sa réalisation et de l'utilisation des nouvelles technologies dans l'art.

Moncef Ben Moussa. Pour répondre, je m'attarderai peut-être aussi sur l'un des services majeurs du musée qui est le service éducatif. Aujourd'hui la Tunisie n'est pas une superpuissance militaire ou économique, mais elle a été visée, en 2015, pour ce que signifie sa culture, pour ce que signifie le Musée national du Bardo. Aujourd'hui, avec les moyens que nous avons, nous sommes incapables de faire face aux esprits qui peuvent commettre un tel acte ignoble. Faire la guerre avec des armes ne suffit pas. Le service éducatif du Bardo accueille les scolaires dans un programme qui leur est consacré.

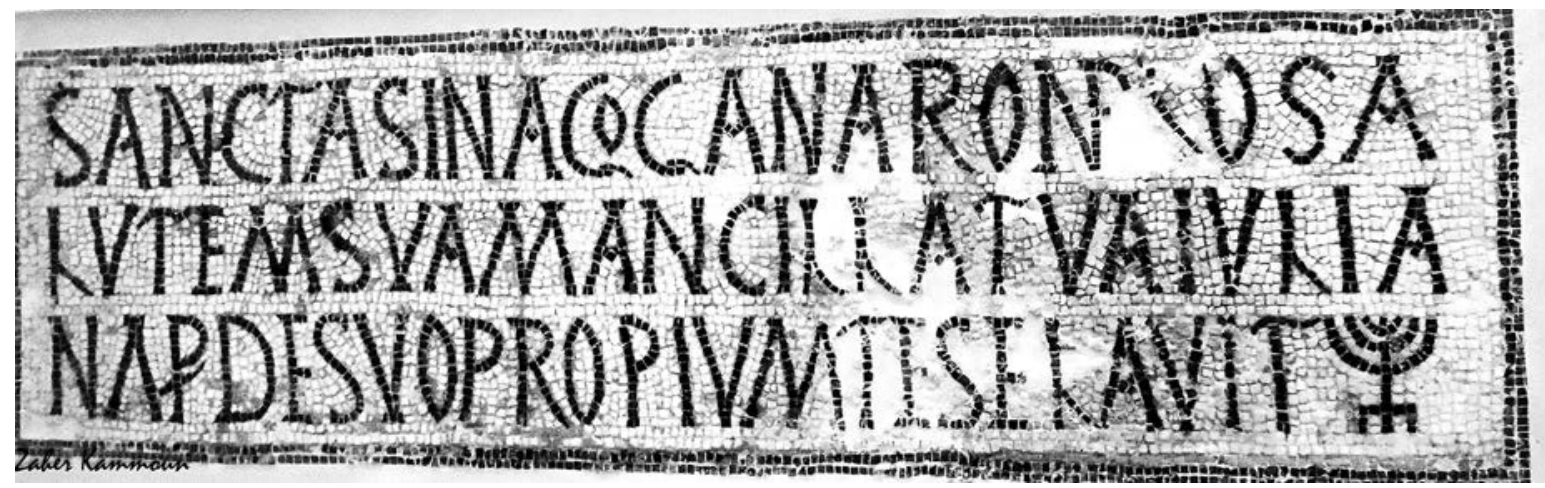


Je pense que quand un élève quitte le Bardo en sachant que les temples païens de tous les sites tunisiens, la synagogue de Hammam Lif (Naro ; fig. 7), les églises qui se trouvent dans presque tous les sites (fig. 8) avec des sédiments de l'antiquité tardive et les mosquées sont des chefs-d'œuvre construits par ses ancêtres, il se doit de s'approprier toutes ces cultures-là, sans distinctions aucunes. $S^{\prime}$ il se dit aujourd'hui musulman, son attitude vis-à-vis des autres cultures, en particulier religieuses, ne doit pas manifester un recul. Je pense que si l'on construit cette idée dans les esprits des jeunes, la dimension universelle ne représente aucune contradiction

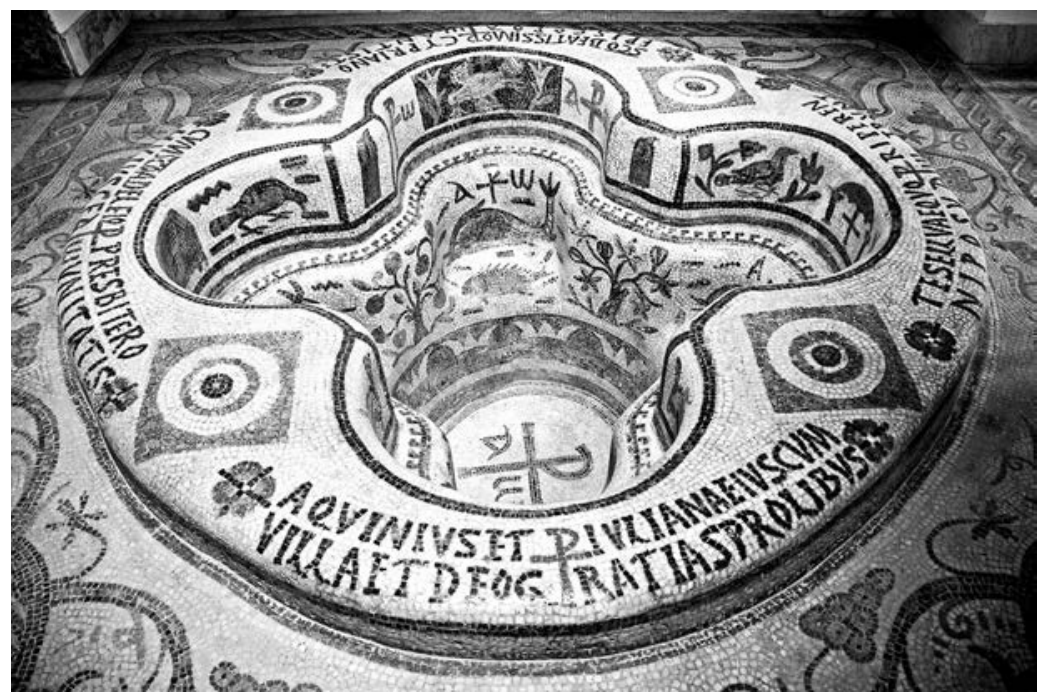
avec le sentiment national.

La politique des institutions de tutelle porte cette double conviction : tout d'abord, la perception même du rôle du musée qui doit faire abstraction des limites temporelles et des limites spatiales. L'héritage culturel n'est nullement sélectif ou il ne doit pas l'être. Il y a parfois, il est vrai, un attachement particulier à une période ou à une autre, c'està-dire à une culture ou à une autre, mais cette attitude n'atteint presque jamais un excès qui nierait le reste. Loin de là, c'est l'acceptation de l'héritage culturel et patrimonial dans sa globalité qui, paradoxalement, renforce un sentiment identitaire profond, tirant sa force de la capacité d'ouverture sur le différent, de la compréhension et de la tolérance. Le Musée national du Bardo a précisément cette particularité de pouvoir refléter de façon exhaustive cette réalité par la présentation d'une image de la Tunisie carrefour de civilisations, ce qui apparaît à travers la diversité extraordinaire de ses collections et une stratification des nombreuses pages d'une longue histoire.

Il va sans dire que l'un des paradoxes que vivent les musées de la rive sud de la Méditerranée est qu'ils sont, d'une part, ouverts aux institutions muséales de la rive nord et du reste du monde, donc intégrés à des réseaux internationaux pour divers projets $^{16}$ et que, d'autre part, ils sont repliés sur eux-mêmes lorsqu'il s'agit de créer un réseau régional pour des projets à court ou à long terme. Il est aussi paradoxal que les musées de la rive sud, du Caire à Nouakchott, n'apparaissent jamais dans I'agenda du Bardo. C'est une sombre réalité, un fait à mettre à notre passif.

Sans prétendre pouvoir faire un état clair de cette difficulté endémique, on peut noter comment, à travers leurs ouvertures aux autres institutions muséales, les musées de la rive sud, sans même s'en rendre compte, suivent la direction de l'immigration. L'explication la plus simple de cette attitude est peut-être tout simplement la conscience d'appartenir au même espace géographique, historique et culturel, attitude dont l'intention globale est de participer ensemble à la construction de ponts entre les deux rives de la Méditerranée.

Malika Dorbani-Bouabdellah. En matière de musée, je ne pense pas que l'on soit héritiers seulement d'un état ou d'un empire, mais aussi d'un idéal politique. Si un pays se dit républicain et démocratique, attaché aux droits de l'homme, si sa devise est « par le peuple et pour le peuple », s'il considère le musée comme aussi important pour le bien-être public que ses autres grandes tâches, ses musées ne peuvent être que l'héritage 


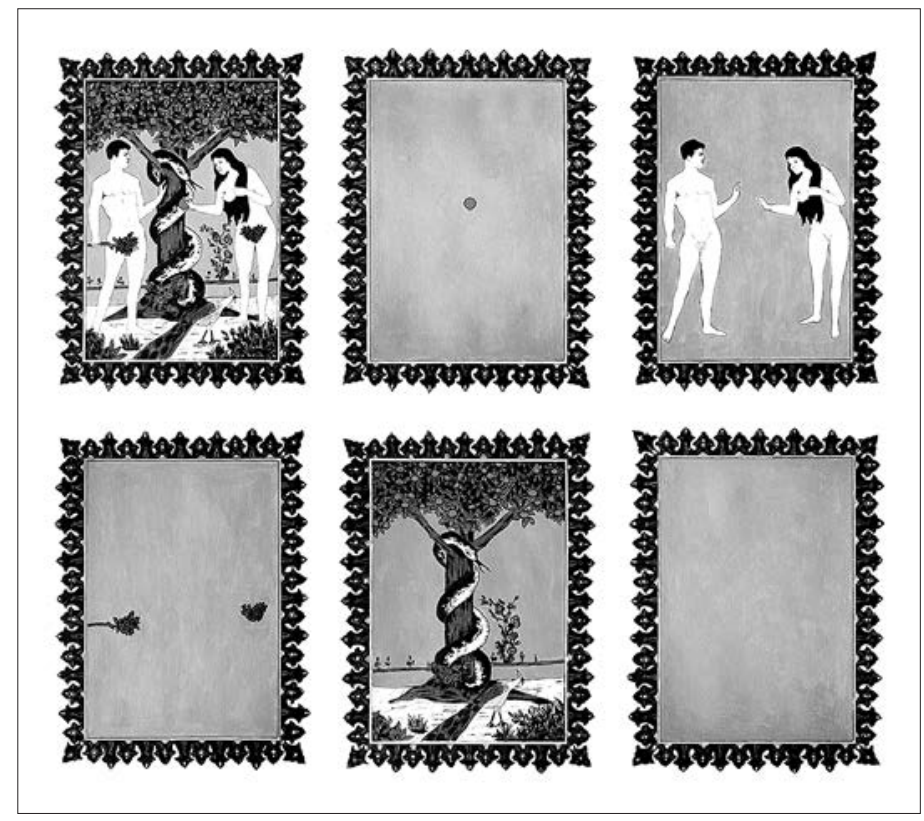

de la république qui en a fait un espace public, laïc, démocratique, universel, consacré à la conservation du patrimoine nationalisé par elle et dont elle s'est fait la dépositaire et la garante. La république en a fait un espace consacré au public citoyen dans un but éducatif et de plaisir esthétique, conformément à l'idée humaniste selon laquelle le peuple possède les vertus de la conscience morale universelle, fondement politique de la démocratie et des droits de I'homme. Dès lors, le musée devient le garant de la protection, de la transmission et de la postérité du patrimoine devenu mémoire collective et richesse nationale. Sans le système républicain qui l'a adopté comme une partie intégrante de son histoire, qui s'est donné le rôle de dépositaire, de gestionnaire et de tuteur des collections qu'il renferme, il n'y aurait pas eu de musée public.

En conservant les musées hérités du temps où ils ne jouissaient pas de leur souveraineté, du temps où ils n'étaient pas concer-

9. Soukaina Joual, Disobey, 2017, série de six miniatures, acrylique sur papier, œuvres exposées pendant «JF JH Égalités » à L'appartement 22, Rabat, février - mai 2017. nés par leur création, en les rénovant et en en créant de nouveaux, les pays du Sud démontrent par les actes qu'ils dépassent le déterminisme historique. Il leur reste à faire des progrès sur les plans démocratique et technologique, à donner à chacun le droit à la jouissance de son patrimoine, à brasser toutes les catégories de publics à des fins de réconciliation et de communion. II reste aussi à appliquer les principes universels qui président à la création et à l'activité d'un musée : la programmation, la conservation, le geste architectural, la muséographie adaptée à la nature des collections et au type de musée, à enseigner l'histoire de l'art, à former et développer les savoir-faire, la profession étant à la fois le métier et la conscience professionnelle. Le musée, par le patrimoine qu'il constitue, conserve et enrichit, par l'éducation qu'il prodigue, par la création qu'il dynamise, par les valeurs, les repères, la mémoire, le lien social qu'il construit, doit être au cœur de la politique et des enjeux sociétaux et mondiaux.

Abdellah Karroum. Pour répondre, je parlerai d'abord d'un autre lieu, L'appartement 22. Ce lieu est né dans un contexte qui n'est pas celui du développement urbain que l'on trouve au Qatar, mais celui du Maroc de 2002, peu après le début de la transition politique qui s'est opérée avec l'ouverture du pays, suite à la mort, quelques années auparavant, de Hassan II. La société civile est alors très active autour du nouveau roi. Le Maroc sort des années de plomb post-indépendance, mais ne possède pas encore d'espace d'expression prêt à accueillir les jeunes artistes et les chercheurs. J'avais donc décidé d'accueillir les artistes de ma génération et le public chez moi, autour d'une table, pour parler des questions qui les préoccupaient. Les premières publications, expositions, débats tournaient autour des questions qui relient les artistes à leur société. La première exposition s'appelait " JF」JH Individualités ${ }^{17}$ ", et dix ans après nous étions parvenus à faire cinq expositions dans la série "JF」JH », sur autant de thèmes que les " concubinages », les " conventions », les « libertés ${ }^{18}$ », les " égalités » (fig. 9)... Ce qui a été très important c'est qu'à chaque fois qu'une exposition était créée à partir de projets artistiques, on voyait un changement dans les textes de lois marocains (le droit des femmes par exemple). 
C'est cela qui m'a permis d'éprouver les enjeux de l'art, mais aussi la responsabilité des artistes et ma responsabilité en tant que commissaire d'exposition. C'est cette notion de citoyenneté, de responsabilité qui m'a conduit à créer ce lieu.

En Afrique, en effet, il y a beaucoup d'initiatives et de collections privées qui sont à l'origine de musées qui deviendront peut-être un jour publics. Le musée existe aussi grâce à des initiatives, une volonté. On observe et on lit toujours la production internationale de l'histoire de l'humanité, de son expression, depuis le lieu où l'on se trouve. Je regrette qu'Abu Dhabi ait choisi de construire un musée du Louvre et un musée Guggenheim ; personnellement, je préfère les situations où la nécessité conduit à créer un musée là où l'on se trouve, c'est pourquoi le musée que je dirige s'appelle le Musée arabe d'art moderne. Ce n'est pas seulement un musée qatari mais un musée créé par les gens du Qatar pour le monde arabe. La majorité des œuvres viennent d'Algérie, de Tunisie, du Maroc, du Soudan, de Turquie, etc., non parce qu'il y a là un point de vue nationaliste, mais bien quelque chose comme une culture commune.

1. Les pages qui suivent offrent une retranscription de la table ronde du 17 novembre 2017, à I'Institut français de Tunis, organisée à l'initiative de I'Institut national d'histoire de l'art à l'occasion de la préparation de son numéro consacré à l'histoire de l'art du et au Maghreb. Nous souhaitons remercier tous ceux qui I'ont rendue possible: Olivier Poivre d'Arvor, ambassadeur de France en Tunisie, Sophie Renaud, directrice de I'Institut français de Tunisie, et Valérie Lesbros, attachée culturelle, tous les intervenants ainsi que l'équipe de la revue, en particulier Marie Caillat.

2. Ce colloque était dirigé par Éric de Chassey, Dominique Jarrassé, Zahia Rahmani et Ahmed Saadaoui, et organisé par I'Académie de France à Rome - Villa Médicis; le Centre F. G. Pariset de l'université Bordeaux-Montaigne; I'École du Louvre, Paris ; avec le soutien de l'École française de Rome ; de l'Institut national d'histoire de l'art, Paris ; de I'Institut de Recherches sur le Maghreb Contemporain, Tunis et du Laboratoire d'Archéologie et d'Architecture Maghrébines de l'université de la Manouba, Tunis. Première session : " L'héritage colonial des musées ", Paris, École du Louvre, 12-13 décembre 2014 ; deuxième session : «Relectures postcoloniales des échanges artistiques et culturels entre Europe et Maghreb (Algérie, France, Italie, Maroc et Tunisie) - XVIII XXI siècles ", Rome, Villa Médicis - Académie de France à Rome, 9-10 avril 2015 ; troisième session (annulée) : "Transferts architecturaux, colonisation archéologique et invention des patrimoines », Tunis, Musée national du Bardo, 4-5 juin 2015.

3. "Déclaration sur l'importance et la valeur des musées universels ", signée par les directeurs des musées suivants : The Art Institute of Chicago ; 
Alte Pinakothek, Neue Pinakothek - Staatlichen Museen, Munich; Staatlichen Museen zu Berlin ; Cleveland Museum of Art ; J. Paul Getty Museum, Los Angeles; Solomon R. Guggenheim Museum, New York ; Los Angeles County Museum of Art ; musée du Louvre, Paris ; The Metropolitan Museum of Art, New York; The Museum of Fine Arts, Boston ; The Museum of Modern Art, New York; Opificio delle Pietre Dure, Florence ; Philadelphia Museum of Art; Museo nacional del Prado, Madrid; Rijksmuseum, Amsterdam ; Musée de l'Ermitage, Saint-Pétersbourg; Museo nacional Thyssen-Bornemisza, Madrid; Whitney Museum of American Art, New York ; The British Museum, Londres[en ligne, en français : http:// icom.museum/fileadmin/user_upload/pdf/ICOM_ News/2004-1/FRE/p4_2004-1.pdf (consulté le 12 décembre 2017)].

4. La $22^{\mathrm{e}}$ conférence des parties à la Conventioncadre des Nations unies sur les changements climatiques a eu lieu à Marrakech du 7 au 18 novembre 2016

5. La rétrospective consacrée à Alberto Giacometti a eu lieu 20 avril au 4 septembre 2016.

6. Présentée du 31 octobre 2017 au 4 février 2018 au MMVI.

7. Organisée dans le cadre du projet «Picasso - Méditerranée 2017-2019 ", initié par le Musée Picasso de Paris, I'exposition «Face à Picasso » a lieu du 17 mai au 31 juillet 2017.

8. Léon L'Africain, "Cour du Roi de Thunes, ordres, cérémonies \& officiers députés en icelle ", dans Description de l'Afrique, tierce partie du monde, contenant les Régions, Royaumes, Villes, Cités, Châteaux et Forteresses..., livre V, Lyon, 1556, p. 279.

9. Sur cette histoire des dons et des donateurs en Algérie, voir la contribution de Bernadette $\mathrm{N}$. Saou-Dufrêne et Amel Djenidi, " Musées, colonialisme, indépendance : figures du donateur », dans ce volume, p. 153-172.

10. Selon I'accord conclu entre les deux pays le 11 juillet 1968, voir "Près de trois cent œuvres d'art ont été restituées par la France ", dans Le Monde du 14 décembre 1969 [en ligne : http:// www.lemonde.fr/archives/article/1969/12/04/ pres-de-trois-cents-uvres-d-art-ont-ete-restituees-par-la-france_2406225_1819218. $\mathrm{html}$ ?xtmc=algerie $\& x t c r=21$ (consulté le 13 décembre 2017)].

11. Dalila Mahammed-Orfali, Arlette Sérullaz et Nathalie Coural (dir), De Delacroix à Matisse. Dessins français du musée des Beaux-Arts d'Alger, cat. exp. (Paris, musée du Louvre, 2003-2004), Paris/ Alger, 2003.

12. Alexandre Kazérouni, Le Miroir des cheikhs. Musée et politique dans les principautés du Golfe persique, Paris, 2017.
13. Okwui Enwezor, "Modernity and Postcolonial Ambivalence ", dans South Atlantic Quarterly, 2010, vol. 109, no 3, p. 595-620 [en ligne, DOI: https://doi.org/10.1215/00382876-2010-008 (consulté le 13 décembre 2017)].

14. "État musulman souverain, attaché à son unité nationale et à son intégrité territoriale, le Royaume du Maroc entend préserver, dans sa plénitude et sa diversité, son identité nationale une et indivisible. Son unité, forgée par la convergence de ses composantes arabo-islamique, amazighe et saharo-hassanie, s'est nourrie et enrichie de ses affluents africain, andalou, hébraïque et méditerranéen. » Préambule de la Constitution marocaine, 2011.

15. Cette sensibilisation passait par leur participation à des ateliers d'une durée de trois à quatre jours autour de la pratique de la sculpture, de la photographie ou de la peinture, animés par un artiste, et incluant une étape de visite des ateliers d'artistes mais aussi de visites guidées d'expositions interactives.

16. L'exposition "Lieux saints partagés " a été présentée au Musée national du Bardo, à Tunis, du 9 novembre 2016 au 12 février 2017, après avoir été montée au Musée national des civilisations de I'Europe et de la Méditerranée, à Marseille, d'avril à août 2015). La version tunisienne, dirigée par Nejib Ben Lazreg (INP), Dionigi Albera (CNRS, IDEMEC/université Aix-Marseille), Manoël Pénicaud (CNRS, IDEMEC/université Aix-Marseille) et Isabelle Marquette (Mucem) est née d'un partenariat entre l'Institut national du Patrimoine, le Musée national du Bardo et le Mucem. Voir Lieux saints partagés, cat. exp. (Marseille, Mucem, 2015), Marseille/Arles, 2015 ; Coexistences. Lieux saints partagés en Europe et en Méditerranée, Dionigi Albera et Manoël Pénicaud (dir.), cat. exp. (Paris, Musée national de l'histoire de l'immigration, 2017-2018), Paris/Arles, 2017.

17. Présentée du 10 octobre au 27 novembre 2002.

18. Le projet «JF_JH Libertés », qui clôturait les dix premières années d'existence de la galerie, s'est déployé sous plusieurs formats, et notamment un programme radio, des conférences et plusieurs expositions (dont une à L'appartement 22, à Rabat, du 24 août au 10 octobre 2012). 